\title{
CDC Grand Rounds: Chronic Fatigue Syndrome - Advancing Research and Clinical Education
}

\author{
Elizabeth R. Unger, PhD, MD ${ }^{1}$; Jin-Mann Sally Lin, $\mathrm{PhD}^{1}$; Dana J. Brimmer PhD ${ }^{1}$; Charles W. Lapp, MD ${ }^{2}$; Anthony L. Komaroff, MD ${ }^{3}$; \\ Avindra Nath, $\mathrm{MD}^{4}$; Susan Laird, $\mathrm{MSN}^{5}$; John Iskander, $\mathrm{MD}^{6}$
}

Chronic fatigue syndrome (CFS) is a complex and serious illness that is often misunderstood. Experts have noted that the terminology "chronic fatigue syndrome" can trivialize this illness and stigmatize persons who experience its symptoms (1). The name was coined by a group of clinicians convened by CDC in the late 1980s to develop a research case definition for the illness, which, at the time, was called chronic Epstein-Barr virus syndrome. The name CFS was suggested because of the characteristic persistent fatigue experienced by all those affected and the evidence that acute or reactivated Epstein-Barr virus infection was not associated with many cases (2). However, the fatigue in this illness is striking and quite distinct from the common fatigue everyone experiences. A variety of other names have been used, including myalgic encephalomyelitis (ME), ME/CFS, chronic fatigue immune dysfunction, and most recently, systemic exertion intolerance disease (3). The lack of agreement about nomenclature need not be an impediment for advancing critically needed research and education. The term ME/CFS will be used in this article.

\section{ME/CFS is a Significant Public Health Problem}

Extrapolating from the three U.S. population-based studies, it is estimated that at least one million persons in the United States suffer from ME/CFS (4-6). These studies indicate that $\mathrm{ME} / \mathrm{CFS}$ is three to four times more common in women than in men. Persons of all racial and ethnic backgrounds are affected; however, the illness is more prevalent in minority and socioeconomically disadvantaged groups. The highest prevalence of illness is in persons aged $40-50$ years, but the age range is broad and includes children and adolescents.

ME/CFS patients, their families, and society all bear significant costs associated with this illness. These include direct medical costs for provider visits and medications and indirect costs of lost productivity. In the United States, the estimated annual cost of lost productivity ranges from 9-37 billion

This is another in a series of occasional MMWR reports titled CDC Grand Rounds. These reports are based on grand rounds presentations at CDC on high-profile issues in public health science, practice, and policy. Information about CDC Grand Rounds is available at http://www.cdc.gov/cdcgrandrounds. dollars, and for direct medical costs, ranges from 9-14 billion dollars, with nearly one quarter of direct medical expenses paid directly by patients and their families (7-9). When ME/CFS occurs in patients aged $<25$ years, these patients might not achieve their full educational potential, resulting in a life-long impact on their earnings ( 7 ).

ME/CFS patients have significant functional impairment as illustrated by findings from CDC's ongoing study of patients in seven clinics of ME/CFS specialists (Figure). Functioning of ME/CFS patients, as measured by subscale scores on the 36-Item Short Form Survey (SF-36), were well below those of healthy persons except for the two subscales reflecting mental and emotional functioning. Despite the severity of their illness, ME/CFS patients face significant barriers to receiving appropriate health care. A population-based study in Georgia found that $55 \%$ of persons with ME/CFS reported at least one barrier to health care; for example, $10 \%$ had financial barriers to seeking needed health care (10). Most persons with ME/ CFS identified in population surveys have been ill $>5$ years and only approximately half continue to seek medical care (4-6). Further, only approximately $20 \%$ received a diagnosis, emphasizing the need for more physician education about this illness.

\section{Clinical Approach to ME/CFS}

There is no "typical" case, but a patient history can be useful in educating physicians about ME/CFS (Box 1). This composite case history illustrates the key features of ME/CFS: significant reduction in ability to perform usual activities accompanied by profound fatigue; significant worsening of symptoms after minimal physical or mental exertion (termed postexertional malaise); unrefreshing sleep; cognitive difficulties; and orthostatic intolerance (such as dizziness and lightheadedness upon standing up). In addition, this patient experienced widespread muscle pain, joint pain, and unpredictable waxing and waning of symptoms. Persons with ME/CFS might be misunderstood because they appear healthy and often have no abnormalities on routine laboratory testing. Clinicians need to be alert to this difficulty and take the time to elicit a good history of the illness, which is critical in the differential diagnosis and can provide evidence of ME/CFS.

Clinical evaluation includes a thorough medical history, psychosocial history, complete physical examination, mental health assessment, and basic laboratory tests to screen for 


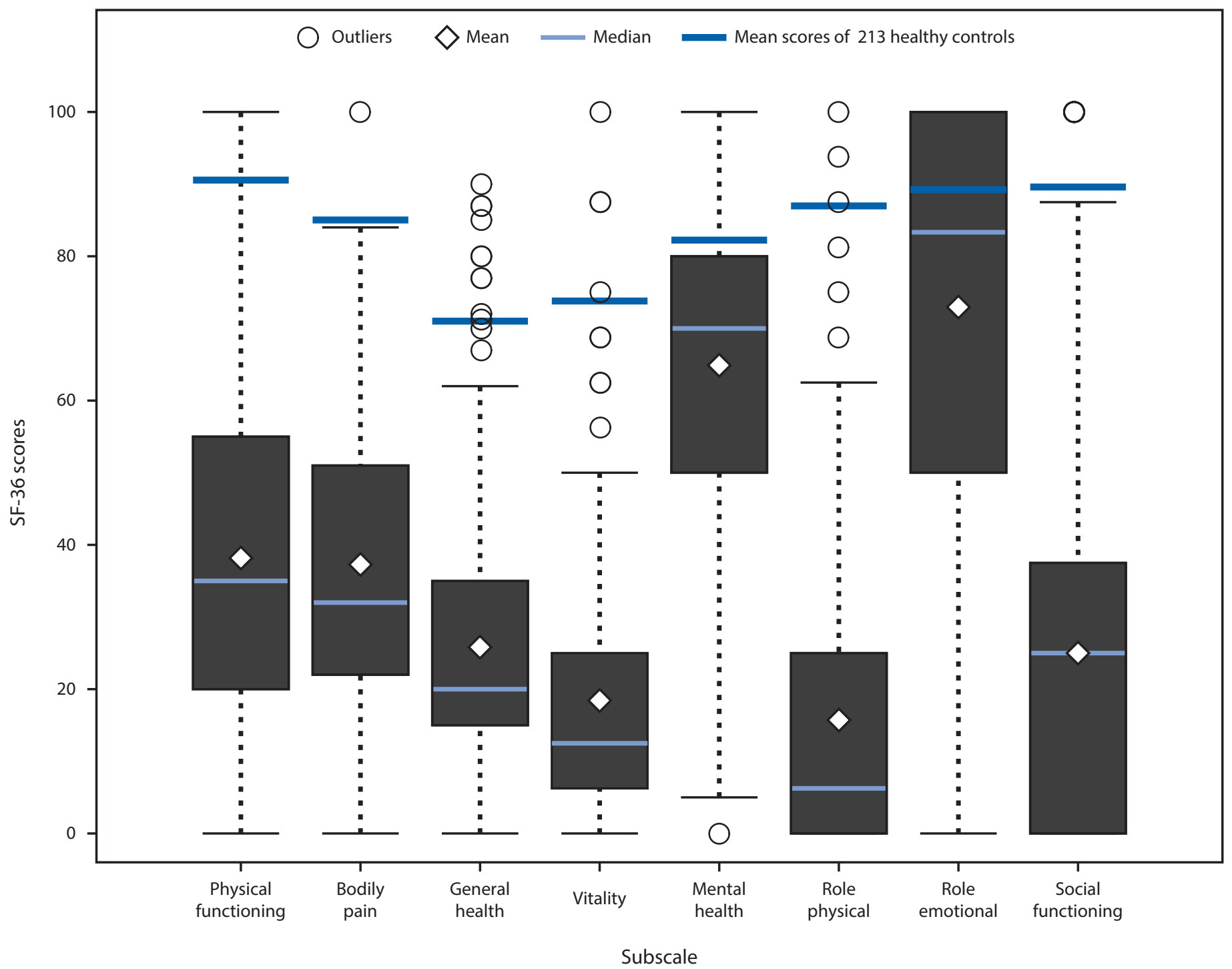

Abbreviations: $\mathrm{CFS}=$ chronic fatigue syndrome; $\mathrm{ME}=$ myalgic encephalomyelitis; SF-36 = 36-Item Short Form Survey.

* Measured by box plots of scores in the eight subscales of SF-36 scores (25th and 75th percentile at bottom and top of box). SF-36 scores range from 0-100, with higher scores indicating better functioning.

† https://www.cdc.gov/cfs/programs/clinical-assessment/index.html.

$\S \mathrm{ME} / \mathrm{CFS}$ patients show significant impairment, particularly in vitality and physical functioning subscale scores, but with preservation of mental health and emotional role functioning.

conditions that could cause symptoms similar to ME/CFS and that should be treated before attributing the illness to ME/CFS. The screening laboratory tests can include complete blood count with differential white blood cell count, sodium, potassium, glucose, blood urea nitrogen, creatinine, lactate dehydrogenase, aspartate transaminase, alanine transaminase, alkaline phosphatase, total protein, albumin, calcium, phosphorus, magnesium, thyroid stimulating hormone, free thyroxine, sedimentation rate, C-reactive protein, antinuclear antibodies, rheumatoid factor, and urinalysis (11). Patients might also have comorbid conditions such as fibromyalgia, irritable bowel and bladder, Sjögren's syndrome, chemical sensitivities, and allergies (11). Additional tests might be clinically indicated.

\section{Cause or Causes of ME/CFS}

The cause or causes of ME/CFS remain unknown. Patients often report an acute onset after a flu-like illness that does not go away, and some patients have a history of frequent infections before their illness. This suggests that an infection can trigger 
the illness, though it is less clear that the ongoing chronic illness is perpetuated by an infection. Investigators have looked for, and failed to find, a single etiologic agent. However, chronic fatiguing illnesses have long been described in the medical literature following infection with several different agents. For example, a syndrome with similarities to ME/CFS occurs in approximately $10 \%$ of patients with a variety of viral and nonviral pathogens, such as Epstein-Barr Virus, Ross River Virus, Coxiella burnetti (Q fever), or Giardia (12). The severity of the acute infection was most predictive of subsequent illness, and there is no evidence of unusual persistence of infections in those who remain ill; baseline psychological profile and socioeconomic status did not predict who would become chronically ill (12). Other studies have found that, compared with healthy controls, persons with ME/CFS have had exposure to significantly more stressors (trauma and other adverse life events) and are more likely to have metabolic syndrome, as well as higher physiologic measures of neuroendocrine response to stress (allostatic load) (13). These associations are not specific to ME/CFS, because stress is a factor in many chronic illnesses. Twin and family studies support the contribution of both genetic and environmental factors in CFS (14). No single mutation or polymorphism has been found that explains most cases of the illness, and a polygenetic explanation for increased susceptibility is most likely.

\section{Treatment of ME/CFS}

At this time, there are no treatments (pharmacologic or nonpharmacologic) that have been proven effective in large randomized trials and replicated by other investigators in other groups of patients with ME/CFS. Recommendations are based on expert clinical opinion and the standard clinical approach to symptom management (15). Sleep disruption and pain are the symptoms usually addressed first, and consultation with sleep or pain management specialists might be helpful. Nonpharmacologic approaches might include Epsom salt soaks, massage, acupuncture, and, most importantly, activity management. Patients should be encouraged to stay active but not too active. They need to start with very low levels of activity and escalate the levels slowly. Brief intervals of activity should be followed by adequate rest to avoid triggering relapse or flare of symptoms, a manifestation of postexertional malaise. Finally, living with a chronic illness is extremely challenging, so attention should be given to addressing depression, anxiety, and improving coping skills.

\section{Addressing ME/CFS}

Recently, three important reports about ME/CFS have been published by authoritative agencies (1). The Institute of
BOX 1. Myalgic encephalomyelitis/chronic fatigue syndrome case history

The patient, aged 37 years, was an internet technologist for a community bank. She had been physically active in sports and working out, and had been maintaining her own household when she experienced a flu-like illness in 2011. She was bedbound at first and slow to recover. Within days she noted an unusual fatigue after minimal activity, then insomnia, achiness in the joints, and generalized muscle pain and weakness. She soon found it difficult to recall recent conversations and events. Reading concentration was limited, and she had trouble comprehending what she had read or even television shows. She would search for words, lose her train of thought, and friends would sometimes have to finish sentences for her. Previously her sleep had always been good, but now she was restless at night and would awaken unrefreshed even after many hours of bed rest. She felt stiff, sore, and foggy for 1-2 hours after awakening. She noted dizziness or lightheadedness on getting up quickly, and on a couple of occasions "saw stars," but did not experience tunnel vision or fainting. The patient was unable to keep up the house, and she had to rely on friends and family to help her with cleaning, laundry, and shopping. She would attempt to keep up at home and at work, but exertion would inevitably make symptoms worse, and if she exerted too much she would end up sick and chairbound for 1-2 days afterward.

Evaluation by her primary care physician revealed low blood pressure, but there was no immediate orthostatic blood pressure drop and otherwise the examination was unremarkable. Blood work was unremarkable. Having no explanation for her symptoms despite the profound reduction in her physical abilities, the patient became anxious about her future and both frustrated and discouraged.

Medicine (IOM) issued a 300-page report in which a panel of physicians and scientists reviewed nearly 9,000 published articles (3). They concluded that ME/CFS is a biologically based illness and proposed a new case definition and name (systemic exertion intolerance). The National Institutes of Health (NIH) held a Pathways to Prevention workshop, drawing similar conclusions about the biology of ME/CFS, and the Agency for Healthcare Research and Quality prepared a review of published literature on diagnosis and treatment $(16,17)$. The IOM panel concluded that "ME/CFS is a serious, chronic, complex systemic disease that often can profoundly affect the lives of patients." Both the IOM and NIH reports conclude that ME/CFS is not primarily a psychological illness, although it might lead to a reactive depression in some patients. 
Although none of the biologic abnormalities identified in ME/ CFS patients are sufficiently sensitive or specific to be used as a diagnostic test, the neurologic and immunologic abnormalities documented emphasize that patients' symptoms are real.

In the absence of a diagnostic test, the IOM report proposes use of a new clinical case definition (Box 2). The new case definition is shorter, easier to apply consistently, and emphasizes that ME/CFS is a diagnosis to be actively made, not simply a diagnosis of exclusion. The IOM report also recommended a new name be considered for the condition: systemic exertion intolerance disease.

It is clear that more basic science research is needed. In September 2015, the NIH intramural program began developing a research protocol to study ME/CFS. The overall hypothesis is that ME/CFS is attributable to an infection that results from immune-mediated brain dysfunction in some patients with acute onset illness. Aim 1 will define the clinical phenotype based on history and physical examination, neurologic assessment, neurocognitive testing, psychiatric evaluation, infectious disease, rheumatologic and neuroendocrine evaluations, and exercise testing. Aim 2 will define the physiologic basis of postexercise fatigue and malaise using functional magnetic resonance imaging, detailed metabolic studies, transcranial magnetic stimulation, and detailed autonomic testing before and after exercise challenge. Aim 3 will determine if there are abnormal immune parameters in the blood and spinal fluid and changes in microbiome profiles. Aim 4 will determine if features of the illness can be reproduced in ex vivo studies using cells or serum from patients and a variety of novel approaches such as induced pluripotent stem cell-derived neurons. Patients will be recruited primarily from well-studied cohorts under the care of clinicians with expertise in diagnosis and management of ME/CFS.

$\mathrm{CDC}$ is continuing its efforts to provide evidence-based information about ME/CFS to health care professionals. In 2012 and 2013, CDC partnered with Medscape to present two roundtable discussions that were targeted to primary care physicians. These reached more than 22,000 physicians and more than 6,000 CME credits were issued. CDC provided free online courses about ME/CFS accredited for both physicians, nurses, and other health care professionals. Because the topic of ME/CFS is rarely covered in medical school courses, CDC initiated a project to develop content for the MedEd Portal, a free online service of peer-reviewed content provided by the Association of American Medical Colleges to medical school faculty. To continue communication with the general public and advocacy community, CDC introduced patientcentered outreach and communication calls. These are 1-hour
BOX 2. Institute of Medicine criteria for diagnosis of myalgic encephalomyelitis/chronic fatigue syndrome

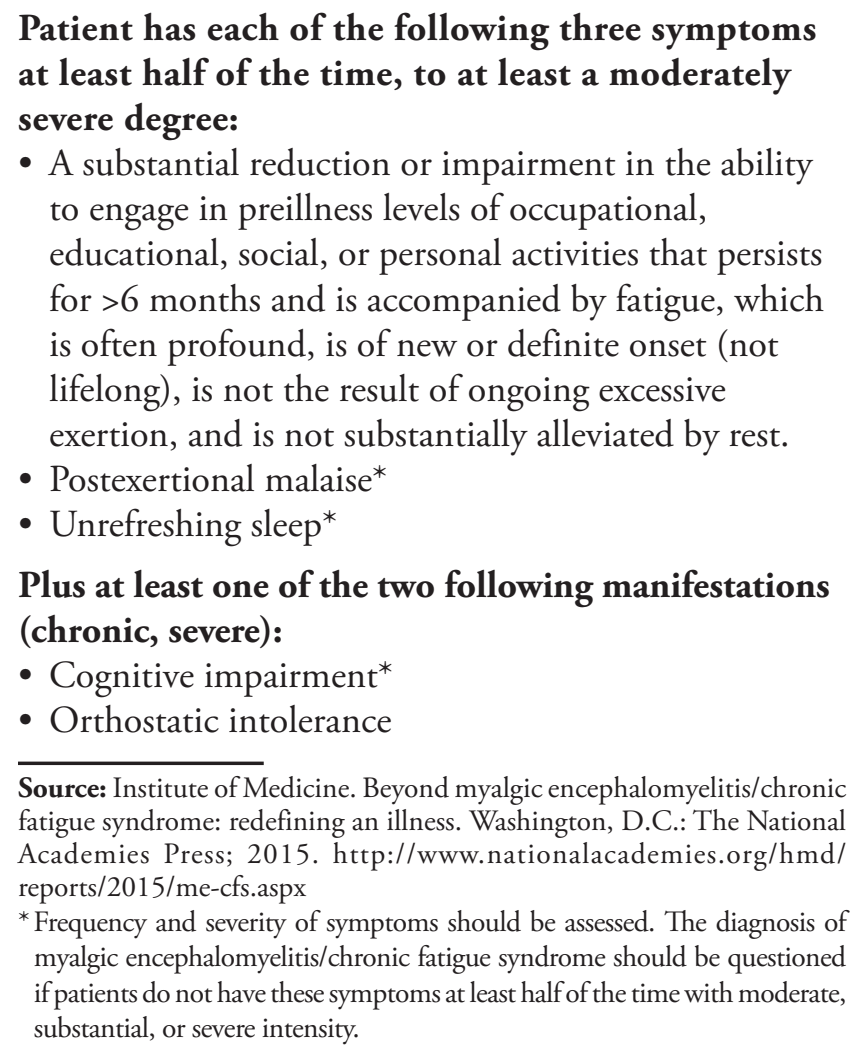

Source: Institute of Medicine. Beyond myalgic encephalomyelitis/chronic fatigue syndrome: redefining an illness. Washington, D.C.: The National Academies Press; 2015. http://www.nationalacademies.org/hmd/ reports/2015/me-cfs.aspx

* Frequency and severity of symptoms should be assessed. The diagnosis of myalgic encephalomyelitis/chronic fatigue syndrome should be questioned if patients do not have these symptoms at least half of the time with moderate, substantial, or severe intensity.

teleconferences held twice a year that are available toll-free in the United States. CDC uses the first 10 minutes to give an update on current activities of the ME/CFS program, and then an outside expert or group of experts presents information on a topic of interest to the community. These are followed by answers to questions submitted to the patient-centered outreach and communication email. Topics have included exercise, infection, and immunity in ME/CFS, ME/CFS and cognitive function, sleep research and ME/CFS, Stanford's research program, and self-management strategies in ME/CFS. Most recently, CDC has begun a new initiative to include broad stakeholder collaboration into developing educational materials. Including the viewpoints of patients, medical professional organizations, medical educators, expert clinicians, and government agencies will help assure the quality and usefulness of these products and facilitate broader dissemination in the medical community. With its demonstrated burden on individual patients and public health, ME/CFS should continue to be an area of active basic science and epidemiologic research, enhanced clinical diagnostic attention and training, and continued outreach, communication, and education. 


\begin{abstract}
${ }^{1}$ Division of High-Consequence Pathogens and Pathology, National Center for Emerging and Zoonotic Diseases, CDC; ${ }^{2}$ Hunter-Hopkins Center, P.A., Charlotte, North Carolina; ${ }^{3}$ Harvard Medical School, and Brigham and Women's Hospital, Harvard University; ${ }^{4}$ Section of Infections of the Nervous System, National Institute of Neurological Diseases and Stroke, National Institutes of Health, Bethesda, Maryland; ${ }^{5}$ Office of the Associate Director for Communication, CDC; ${ }^{6}$ Office of the Associate Director for Science, CDC.
\end{abstract}

Corresponding author: Elizabeth R. Unger, eunger@cdc.gov, 404-639-3533.

\section{References}

1. Komaroff AL. Myalgic encephalomyelitis/chronic fatigue syndrome: a real illness. Ann Intern Med 2015;162:871-2. http://dx.doi. org/10.7326/M15-0647

2. Holmes GP, Kaplan JE, Gantz NM, et al. Chronic fatigue syndrome: a working case definition. Ann Intern Med 1988;108:387-9. http:// dx.doi.org/10.7326/0003-4819-108-3-387

3. Institute of Medicine. Beyond myalgic encephalomyelitis/chronic fatigue syndrome: redefining an illness. Washington, DC: The National Academies Press; 2015. http://www.nationalacademies.org/hmd/ reports $/ 2015 /$ me-cfs.aspx

4. Jason LA, Richman JA, Rademaker AW, et al. A community-based study of chronic fatigue syndrome. Arch Intern Med 1999;159:2129-37. http://dx.doi.org/10.1001/archinte.159.18.2129

5. Reyes M, Nisenbaum R, Hoaglin DC, et al. Prevalence and incidence of chronic fatigue syndrome in Wichita, Kansas. Arch Intern Med 2003;163:1530-6. http://dx.doi.org/10.1001/archinte.163.13.1530

6. Reeves WC, Jones JF, Maloney E, et al. Prevalence of chronic fatigue syndrome in metropolitan, urban, and rural Georgia. Popul Health Metr 2007;5:5. http://dx.doi.org/10.1186/1478-7954-5-5

7. Lin JM, Resch SC, Brimmer DJ, et al. The economic impact of chronic fatigue syndrome in Georgia: direct and indirect costs. Cost Eff Resour Alloc 2011;9:1. http://dx.doi.org/10.1186/1478-7547-9-1

8. Reynolds KJ, Vernon SD, Bouchery E, Reeves WC. The economic impact of chronic fatigue syndrome. Cost Eff Resour Alloc 2004;2:4. http://dx.doi.org/10.1186/1478-7547-2-4
9. Jason LA, Benton MC, Valentine L, Johnson A, Torres-Harding S. The economic impact of ME/CFS: individual and societal costs. Dyn Med 2008;7:6. http://dx.doi.org/10.1186/1476-5918-7-6

10. Lin JM, Brimmer DJ, Boneva RS, Jones JF, Reeves WC. Barriers to healthcare utilization in fatiguing illness: a population-based study in Georgia. BMC Health Serv Res 2009;9:13. http://dx.doi. org/10.1186/1472-6963-9-13

11. Fukuda K, Straus SE, Hickie I, Sharpe MC, Dobbins JG, Komaroff A; International Chronic Fatigue Syndrome Study Group. The chronic fatigue syndrome: a comprehensive approach to its definition and study. Ann Intern Med 1994;121:953-9. http://dx.doi. org/10.7326/0003-4819-121-12-199412150-00009

12. Hickie I, Davenport T, Wakefield D, et al.; Dubbo Infection Outcomes Study Group. Post-infective and chronic fatigue syndromes precipitated by viral and non-viral pathogens: prospective cohort study. BMJ 2006;333:575. http://dx.doi.org/10.1136/bmj.38933.585764.AE

13. Maloney EM, Boneva RS, Lin JMS, Reeves WC. Chronic fatigue syndrome is associated with metabolic syndrome: results from a casecontrol study in Georgia. Metabolism 2010;59:1351-7. http://dx.doi. org/10.1016/j.metabol.2009.12.019

14. Buchwald D, Herrell R, Ashton S, et al. A twin study of chronic fatigue. Psychosom Med 2001;63:936-43.

15. International Association for Chronic Fatigue Syndrome/Myalgic Encephalomyelitis. Chronic fatigue syndrome/myalgic encephalomyelitis primer for clinical practitioners. Bethesda, MD: International Association for Chronic Fatigue Syndrome/Myalgic Encephalomyelitis; 2014. http:// iacfsme.org/portals/0/pdf/Primer_Post_2014_conference.pdf

16. Green CR, Cowan P, Elk R, O’Neil KM, Rasmussen AL. National Institutes of Health Pathways to Prevention Workshop: advancing the research on myalgic encephalomyelitis/chronic fatigue syndrome. Ann Intern Med 2015;162:860-5. http://dx.doi.org/10.7326/M15-0338

17. Haney E, Smith MEB, McDonagh M, et al. Diagnostic methods for myalgic encephalomyelitis/chronic fatigue syndrome: a systematic review for a National Institutes of Health Pathways to Prevention Workshop. Ann Intern Med 2015;162:834-40. http://dx.doi.org/10.7326/M15-0443 